\title{
Adoção de Software de Código Aberto: Uma Revisão Sistemática da Literatura
}

\author{
Luciana Guimarães Carvalho $^{12}$, Fernando Silva Parreiras ${ }^{1}$ \\ ${ }^{1}$ Laboratório de Sistemas de Informação Avançados (LAIS), Universidade FUMEC \\ Av. Afonso Pena, no 3880 - 30.130-009 - Belo Horizonte - MG - Brasil \\ ${ }^{2}$ Universidade Federal de Minas Gerais (UFMG) \\ Av. Antônio Carlos 6627 - 31.270-901 - Belo Horizonte - MG - Brasil \\ lugcarvalho@fumec.edu.br, fernando.parreiras@fumec.br
}

\begin{abstract}
The movement of open source software (OSS) has changed the basic nature of the software industry, and today the use of OSS is reality in business. This article aims to identify what has been reported in the literature that discuss the use of OSS in companies seeking to characterize factors, forms of adoption and business models applied. The method adopted was the systematic literature review and, as search tool we used Google Scholar. As a result we obtained 137 publications with distribution by topic: $51 \%$ discussed factors, $38 \%$ ways of adoption and $11 \%$ business models. This article contributes to the definition of strategies for adoption of OSS by consolidating characteristics identified in previous studies.
\end{abstract}

Resumo. O movimento do software de código aberto (SCA) mudou a natureza básica da indústria de software e, hoje, o uso de SCA é realidade nas empresas. Este artigo objetiva identificar o que foi discutido nas publicações que abordam a utilização do SCA em empresas, buscando identificar fatores, formas de adoção e modelos de negócio aplicados. O método adotado foi a revisão sistemática da literatura e como ferramenta de busca utilizou-se o Google Acadêmico. Como resultado obteve-se 137 publicações sendo a distribuição por tema: $51 \%$ abordaram fatores, $38 \%$ formas de adoção e $11 \%$ modelos de negócio. Este artigo contribui para definição de estratégias para adoção de SCA por consolidar características identificadas em estudos anteriores.

\section{Introdução}

As empresas se apoiam em tecnologias da informação e softwares para executar suas operações e, com isso, visam a eficiência, o aumento de produtividade e de competitividade [Goode 2005]. O advento do movimento do software de código aberto (SCA) 1 mudou a natureza básica da indústria de software [Fitzgerald 2006]. O SCA alterou a forma de desenvolvimento, aquisição, utilização e comercialização de softwares [Ayala et al. 2011] e as organizações de todos os segmentos foram atingidas de alguma forma.

\footnotetext{
${ }^{1}$ No âmbito deste artigo, o termo Software de Código Aberto (SCA) representa também software livre (SL) e software livre e de código aberto (SLCA), exceto quando houver detalhamento de alguma especificidade. Também estão representadas as versões em língua estrangeira, Open source software (OSS), Freellibre open source software (FLOSS) e Free software (FS).
} 
O SCA começou limitado à infraestrutura e agora também é aceito nas camadas mais altas, que são os aplicativos para usuários [Glynn et al. 2005]. O movimento ganha força em resposta à dependência de fornecedor (lock-in), muito presente no modelo tradicional de software proprietário (SP) [Zhu and Zhou 2011], e pela importância em se ter controle e liberdade de escolha [West et al. 2005]. A importância do SCA pode ser evidenciada com o ingresso de grandes empresas como IBM e HP [Theunissen et al. 2004, Richter et al. 2009], fornecedores comerciais com capacidade financeira para assumir compromissos dos produtos de SCA [Holck et al. 2005].

Apesar do aumento do uso de SCA pelas empresas, o número de pesquisas acadêmicas tem diminuído nos últimos 5 anos. Dentre as publicações analisadas nesta revisão foram encontrados temas como: políticas públicas, custos de produto, custos de implantação, ideologia, metodologia de desenvolvimento, comunidade, adoção por desenvolvedores e adoção por empresas.

Esta revisão sistemática tem como objetivo mapear na literatura acadêmica publicações relacionadas a utilização de software de código aberto por empresas, com finalidade de identificar os fatores motivadores e inibidores, formas de adoção e modelos de negócio aplicados.

Este artigo está estruturado em quatro seções. A seção 1, que é a presente introdução. Na seção 2, uma breve consideração sobre software de código aberto e sua adoção. Em seguida, na seção 3, está a descrição do processo da revisão sistemática de literatura até apresentação dos resultados. Na seção 4, estão os trabalhos relacionados. Encerrando, na seção 5, com a conclusão.

\section{Software de Código Aberto}

Segundo [Stallman 1999], o software de código aberto existe desde o início da informática. Dos anos 1960 a meados de 1970, o software não tinha valor comercial pois o foco do mercado era o hardware. A partir da segunda metade de 1970, começou a comercialização de licenças de software e, consequentemente, o "fechamento" do código fonte, dando início ao software proprietário (SP).

O SP é aquele tratado comercialmente como um bem de propriedade intelectual, protegido por copyright e licenças. Outra característica marcante do SP é que seu código fonte $^{2}$ não é disponibilizado ou acessível ao usuário. Já no software de código aberto (SCA), o código fonte é aberto e distribuído, permitindo aos usuários estudá-lo e modificá-lo para atender as necessidades individuais [Rusovan et al. 2005].

Para [West 2003] o movimento do software de código aberto teve sucesso por três fatores principais: necessidade dos usuários por um sistema operacional similar ao Unix porém livre das restrições impostas pela licença da $\mathrm{AT} \& \mathrm{~T}^{3}$; movimento filosófico rejeitando a ideia de dono e propriedade do software; e, por último, a popularização da Internet facilitando a comunicação e o compartilhamento de informações.

\subsection{Adoção de software de código aberto}

Para [Holck et al. 2005], SCA se tornou uma importante alternativa perante ao SP quando as organizações decidem sobre aquisição de software.

\footnotetext{
${ }^{2}$ Código fonte são os arquivos que contêm as instruções para o computador executar determinada função.

${ }^{3}$ AT\&T desenvolveu o Unix na década de 1970
} 
Em pesquisa realizada na União Europeia por [Wichmann 2002], investigou-se a utilização do SCA pelas empresas, agrupando os diversos softwares em quatro áreas de uso: sistema operacional de servidores, banco de dados, SCA em desktops e SCA envolvido na criação ou operacionalização de sites. Foi solicitado aos estabelecimentos pesquisados que indicassem, para cada um dos critérios, o grau de importância nas últimas decisões de usar SCA em vez de SP. O resultado encontrado para sistema operacional de servidores foi que 56\% das empresas pesquisadas usam SCA ou pretendem usar, e os fatores mais importantes foram: maior estabilidade e melhor controle de acesso. Quanto ao banco de dados, $42 \%$ das empresas usam ou planejam usar banco de dados SCA e os fatores considerados mais importantes foram: maior estabilidade e melhor controle de acesso. Apenas $20 \%$ das empresas usam ou pretendem usar algum SCA no desktop e os fatores mais importantes foram: maior estabilidade e menor custo com licença. 39\% das empresas que participaram da pesquisa revelaram que usam ou pretendem usar SCA para criação ou operacionalização de sites, e os fatores considerados mais importantes para adoção foram: maior estabilidade e melhor preço em relação ao desempenho.

No levantamento realizado por [Softex 2005], as principais motivações para adoção de SCA identificadas pelos usuários foram a redução de custos e o desenvolvimento de novas habilidades. Com menor frequência, surgiram fatores como facilidade das ferramentas e razões ideológicas (por exemplo: "software não deve ser proprietário" e limitar o poder das grandes corporações).

Ainda no levantamento realizado por [Softex 2005], foi identificada convergência sobre a intensidade do uso de SCA em alguns setores econômicos caracterizando três tipos principais de mercados: a) TIC, berço natural do SCA, com desenvolvimentos tanto de sistemas operacionais, infraestrutura, middleware e aplicativos; b) Governo, provocada por razões filosóficas e de suposta redução de custos, incluindo principalmente sistemas operacionais mas também infraestrutura, middleware e aplicativos; c) Serviços (comércio e educação e, em menor frequência, a área de saúde), com forte presença de sistemas operacionais, secundariamente outros itens de infraestrutura, e pouca presença de aplicativos para usuário.

\section{Revisão Sistemática da Literatura}

Para identificar o que se sabe sobre a utilização de SCA pelas empresas, realizouse uma revisão sistemática da literatura (RSL), seguindo as diretrizes propostas por [Kitchenham 2004]. Revisão sistemática da literatura é definida "como um meio de identificar, avaliar e interpretar todas as pesquisas disponíveis relevantes para a questão de pesquisa específica ou área temática ou fenômeno de interesse" [Kitchenham 2004].

A principal característica que diferencia uma revisão sistemática de uma revisão da literatura é a definição do protocolo de avaliação. Os dados coletados a partir da RSL são pré-requisito para a meta-análise quantitativa [Kitchenham 2004]. O protocolo é composto a partir da definição de critérios, sendo eles:

- critérios de busca

- critérios de inclusão

- critérios de exclusão

- definição dos dados que serão extraídos e analisados

- critérios de qualidade 
Os três momentos da revisão sistemática definidos por [Kitchenham 2004] são: planejamento, realização e apresentação dos resultados.

\subsection{Planejamento}

No planejamento, é o momento de identificar a necessidade da revisão e desenvolver um protocolo de avaliação [Kitchenham 2004].

Nesta revisão sistemática de literatura definiu-se como objetivo responder as seguintes questões:

Q1 Quais os fatores motivadores e inibidores encontrados pela empresas ao adotarem SCA?

Q2 Como as empresas usam SCA? Quais são as formas de adoção de SCA?

Q3 Quais os modelos de negócios, que envolve SCA, são praticados pelas empresas?

A tabela 1 apresenta o protocolo de avaliação utilizado.

Tabela 1: Protocolo da revisão sistemática de literatura

\begin{tabular}{|l|l|}
\hline Ferramenta de busca & Google Acadêmico \\
\hline Critérios de busca & $\begin{array}{l}\text { OSS adoption } \\
\text { open source software adoption } \\
\text { open source adoption } \\
\text { adopt open source } \\
\text { OSS use } \\
\text { open source software use } \\
\text { open source software usage } \\
\text { open source use } \\
\text { open source usage } \\
\text { free software adoption } \\
\text { free software use } \\
\text { free software usage } \\
\text { adoção de software livre } \\
\text { adoção do software livre } \\
\text { adoção de software aberto } \\
\text { adoção do software aberto } \\
\text { adoção de software de código aberto } \\
\text { adoção do software de código aberto } \\
\text { uso de software livre } \\
\text { uso do software livre } \\
\text { uso de software aberto } \\
\text { uso de software aberto } \\
\text { uso de software de código aberto } \\
\text { uso do software de código aberto } \\
\text { artigos publicado em revistas científicas ou anais de } \\
\text { congressos } \\
\text { teses e dissertações defendidas } \\
\text { relatório de pesquisa de entidade idônea } \\
\text { capítulos de livros }\end{array}$ \\
\hline Critérios de inclusão \\
\end{tabular}




\begin{tabular}{|l|l|}
\hline Critérios de exclusão & $\begin{array}{l}\text { não disponibilizar o texto completo para leitura } \\
\text { não abordar o tema adoção/uso de software de código } \\
\text { aberto em empresas } \\
\text { não abordar os temas fatores, formas de adoção ou } \\
\text { modelo de negócio }\end{array}$ \\
\hline Dados coletados & $\begin{array}{l}\text { ano de publicação } \\
\text { tipo de publicação } \\
\text { título da publicação } \\
\text { autores } \\
\text { questão/objetivo da pesquisa } \\
\text { método de pesquisa } \\
\text { palavras-chave } \\
\text { contexto organizacional } \\
\text { fatores motivadores } \\
\text { fatores inibidores } \\
\text { categoria de software estudada } \\
\text { forma de adoção } \\
\text { modelo de negócio }\end{array}$ \\
\hline Critérios de qualidade & O método de pesquisa está explícito? \\
A questão de pesquisa e objetivo estão explícitos ? & O contexto da pesquisa está descrito ? \\
Está descrito como foi feita a coleta de dados ? \\
Está descrito como os dados foram analisados ?
\end{tabular}

As etapas indicadas no protocolo não são sequenciais, envolvem iterações. As atividades são iniciadas durante a fase de desenvolvimento do protocolo e são refinadas enquanto a revisão ocorre. [Kitchenham 2004] cita exemplos :

- A seleção dos estudos primários se rege por critérios de inclusão e exclusão. Estes critérios são inicialmente especificados na definição do protocolo, mas podem ser refinados a medida em que os critérios de qualidade são definidos.

- A forma de extração de dados, ou critérios de busca, é preparada durante a construção do protocolo e alterada quando são definidos os critérios de qualidade.

- Os parâmetros para síntese de dados definidos inicialmente no protocolo podem ser alterados na medida em que os dados são coletados.

\subsection{Realização}

Após o planejamento da revisão sistemática, começa o processo de seleção e filtragem das publicações conforme os critérios estabelecidos no protocolo.

Na etapa de realização, cinco estágios são necessários: identificação da pesquisa, seleção dos estudos primários, avaliação da qualidade dos estudos coletados, extração e monitoração de dados, e síntese dos dados [Kitchenham 2004]. 


\section{Identificação da pesquisa}

Nesse estágio, os critérios de busca são testados e refinados até que se tenha um conjunto de publicações satisfatório para a pesquisa. A ferramenta de busca Google Scholar foi escolhida por ter amplo acesso a várias fontes de publicações. O critério de busca foi codificado de forma que a ferramenta procurasse quaisquer dos termos listados no critério de busca do protocolo. A ferramenta retornou 14.800 itens.

\section{Seleção dos estudos primários}

A seleção dos estudos primários foi feita através da leitura dos títulos e resumos das publicações apresentadas como resultado da busca do estágio anterior.

A ferramenta de busca utilizada apresenta os resultados em ordem de relevância dos critérios informados. A partir do resultado de número 470, as publicações apresentadas já não estavam aderentes aos critérios de busca e optou-se por interromper o processo de seleção.

Em seguida, foram aplicados os critérios de inclusão e exclusão. As publicações selecionadas foram catalogadas usando a ferramenta Mendeley Desktop ${ }^{4}$.

\section{Avaliação da qualidade dos estudos coletados}

A avaliação das publicações selecionadas no estágio anterior foi realizada aplicando os critérios de qualidade. Como resultado, 137 publicações seguiram para o próximo estágio.

\section{Extração e monitoração de dados}

Nesse estágio, as publicações são inteiramente lidas e os dados são coletados. O Mendeley e um software de planilha eletrônica foram usados para armazenar os dados coletados, preparando-os para o próximo estágio.

\section{Síntese dos dados}

As publicações foram classificadas por tema abordado, por ano, por tipo. Também foram identificados: o contexto da pesquisa; se foi aplicado algum modelo de aceitação de tecnologia; quais fatores foram relacionados nos resultados; se foi citado alguma forma de adoção ou modelo de negócio envolvendo SCA. A classificação por tema abordado considerou as informações apresentadas com maior relevância na seção de resultados das publicações, visto que houveram casos onde foi abordado mais de um tema.

\subsection{Resultados}

O último momento da revisão é a elaboração do texto final, onde todas as informações usadas para coletar os estudos será exposta juntamente com os resultados encontrados,

\footnotetext{
${ }^{4}$ Mendeley Desktop é um software para gerenciamento de referências que armazena metadados das publicações podendo inclusive associar arquivos em diversos formatos à referência.
} 
com as devidas análises conforme o objetivo da pesquisa [Kitchenham 2004].

Como resultado da revisão sistemática de literatura obteve-se 137 publicações selecionadas, sendo que 70 abordaram sobre os fatores considerados no processo de adoção, 52 investigaram sobre a forma de adoção de SCA pelas empresas e 15 estudaram sobre modelo de negócio praticado que envolve SCA. A figura 1 apresenta a proporcionalidade dos temas objetivo dessa revisão sistemática de literatura, em relação às publicações analisadas ao longo dos anos.

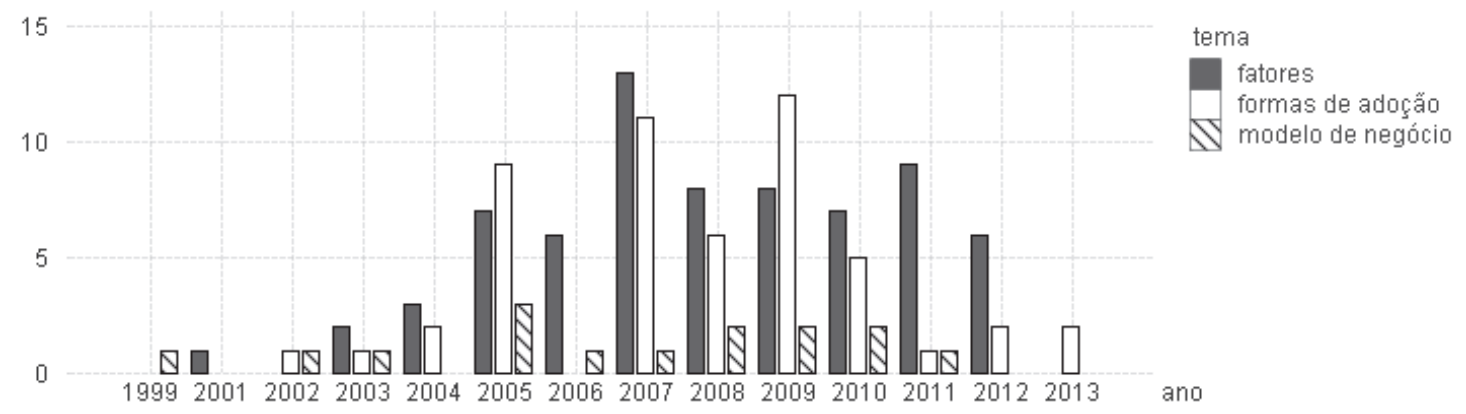

Figura 1. Ocorrência dos temas por ano

\section{Q1 Fatores motivadores e inibidores}

Os fatores motivadores e inibidores mais citados nas publicações selecionadas estão listados nas tabelas 2 e 3, apresentados em ordem decrescente da quantidade de publicações em que foi relacionado como resultado da pesquisa.

Tabela 2. Fatores motivadores mais citados na revisão sistemática de literatura
\begin{tabular}{l|l|}
\hline Fator motivador & $\begin{array}{l}\text { Número de } \\
\text { publicações }\end{array}$ \\
\hline Redução de custos & 39 \\
\hline Independência de fornecedor & 21 \\
\hline Segurança & 16 \\
\hline Qualidade & 12 \\
\hline Acesso ao código fonte & 11 \\
\hline Confiabilidade & 9 \\
\hline Performance & 8 \\
\hline Interoperabilidade & 7 \\
\hline Permitir customização & 6 \\
\hline
\end{tabular}

\section{Q2 Formas de adoção}

Na revisão sistemática de literatura, os artigos que abordaram forma de adoção discutiram como as empresas adotaram SCA, não havendo discussão sobre as várias opções de adoção ou sobre a influência de fatores nas diferentes formas de adoção. O contexto era empresa - pública ou privada, de setores como: indústria, saúde, educação e 
Tabela 3. Fatores inibidores mais citados na revisão sistemática de literatura

\begin{tabular}{|l|l|}
\hline Fator inibidor & $\begin{array}{l}\text { Número de } \\
\text { publicações }\end{array}$ \\
\hline Falta de suporte & 19 \\
\hline Incompatibilidade & 17 \\
\hline Falta de conhecimento & 9 \\
\hline Escassez de mão de obra técnica & 8 \\
\hline Achar que SCA é mais difícil de usar & 7 \\
\hline Custos de migração & 5 \\
\hline
\end{tabular}

administração pública - sendo abordados assuntos como: plano de migração; direcionamento e influência de políticas públicas; formas de customização de SCA para uso interno; e análise quantitativa de quais SCA são usados em determinado setor, cidade, até mesmo país. As publicações que discutiram opções de formas de adoção, variando de acordo com a necessidade ou condições da empresa, tiveram como contexto empresas de tecnologia.

As 52 publicações que abordaram formas de adoção de SCA foram classificadas conforme as categorias identificadas pelos estudos de [Ayala et al. 2011, Ziemer et al. 2008, Höst and Oručević-Alagić 2011]. A tabela 4 apresenta o número de publicações que abordaram cada uma das formas de adoção.

Tabela 4. Formas de adoção de SCA citadas na revisão sistemática de literatura

\begin{tabular}{|l|l|}
\hline Forma de adoção & $\begin{array}{l}\text { Número de } \\
\text { publicações }\end{array}$ \\
\hline Uso de SCA & 43 \\
\hline Uso de SCA para desenvolvimento de software & 13 \\
\hline Uso de componentes SCA & 11 \\
\hline $\begin{array}{l}\text { Uso de práticas e ferramentas praticados pela comu- } \\
\text { nidade de SCA }\end{array}$ & 7 \\
\hline Participação em comunidades de SCA & 7 \\
\hline Desenvolvimento ou provimento de produto SCA & 9 \\
\hline
\end{tabular}

\section{Q3 Modelos de negócio}

Para [Raymond 1999], o SCA pode ser usado como fonte de negócio; cita alguns exemplos: como base para produção de um produto proprietário; consultoria técnica para implantação e manutenção; e uso embarcado em dispositivos de hardware. Existem diversos modelos de negócios envolvendo SCA, desde treinamento, consultoria, serviços de integração e distribuição, a outros mais dinâmicos e inovadores [Hecker 1999].

Para [Dipold 2005], no modelo de negócio que envolve SCA o foco passa a ser o relacionamento com o cliente e não o produto de software em si. Para atender demandas empresariais surgiu um modelo de negócio onde o fornecedor é um intermediário entre empresas e comunidades, oferecendo suporte com níveis de serviço similar aos ofertados 
nos negócios tradicionais da indústria de software. A diferença é que no modelo SCA o processo de aquisição é iniciado pelo próprio usuário - que tem liberdade de baixar o software e testá-lo, sem interveniência do fornecedor - onde a contratação do fornecedor é uma opção, e não obrigatória como no software proprietário com a compra da licença de uso [Taurion 2004].

Na pesquisa de [da Silva et al. 2005] foram detectados cinco tipos de empresas atuando em SCA no Brasil: a) micros e pequenas empresas fundadas nas décadas de 1980 e 1990, dedicadas principalmente ao software proprietário mas que entraram no SCA por exigências do mercado; b) micros e pequenas empresas de fundação mais recente que têm grande parte de suas atividades em SCA e já sobrevivem nesses modelos de negócio; c) grandes empresas, algumas delas multinacionais, que também ingressaram no mundo de SCA, disponibilizando seus produtos proprietários para rodar nesta plataforma e oferecendo suporte pré e pós vendas; d) empresas públicas atuando principalmente na migração da infraestrutura administrativa; e) empresas de software embarcado, que conseguiram tornar seus equipamentos competitivos e mais adaptados com o uso de SCA.

O estudo feito na Europa pelo instituto Berlecon Research [FLOSS 2002] apresenta modelos de negócios envolvendo SCA. As empresas do mercado de serviços relacionados com SCA diferem de acordo com o seu plano de atuação. Primeiramente, existem empresas que têm experiência em outros produtos SCA e têm como principal competência o conhecimento tecnológico e do produto em si, por isso oferecem uma gama completa de serviços. Em segundo lugar estão as empresas que têm conhecimento do processo e prestam serviço relacionado à TI em geral, como consultoria, integração de sistemas, treinamento ou recrutamento de pessoal, às vezes até com certa especialização funcional ou específica.

Foram 15 as publicações que abordaram sobre modelos de negócio envolvendo SCA. O assunto estratégia para modelo de negócio foi abordado por 4 publicações, apenas uma estudou o impacto no negócio quando do uso de SCA embarcado em produtos. A tabela 5 apresenta a quantidade de publicações que identificaram cada um dos modelos relacionados por [FLOSS 2002].

Tabela 5. Modelos de negócios citados na revisão sistemática de literatura

\begin{tabular}{|l|l|}
\hline Modelo de negócio & $\begin{array}{l}\text { Número de } \\
\text { publicações }\end{array}$ \\
\hline Distribuidores de sistema operacional & 3 \\
\hline Distribuidores de SCA especialista & 4 \\
\hline $\begin{array}{l}\text { Distribuidores varejistas de SCA e produtos comple- } \\
\text { mentares }\end{array}$ & 1 \\
\hline Serviços de desenvolvimento / customização de SCA & 7 \\
\hline Provedores de serviços e de suporte & 6 \\
\hline
\end{tabular}

\section{Trabalhos Relacionados}

Durante essa revisão não foram encontradas publicações com a mesma abordagem aqui realizada, porém cinco publicações usaram a revisão de literatura como metodologia. 
[Hauge et al. 2010, Ayala et al. 2011] são duas publicações obtidas da mesma revisão sistemática de literatura, porém apresentadas com foco de análise diferenciado. [Hauge et al. 2010] focou no processo da revisão sistemática e apresentou resultados bibliométricos e análises sobre as formas de adoção de SCA nas empresas. [Ayala et al. 2011] não apresentou detalhes da revisão e focou em questões que devem ser consideradas por empresas que desejam adotar SCA. [Stol and Ali Babar 2010] analisaram o uso de componentes com código aberto no desenvolvimento de softwares. Diferente das publicações citadas, este artigo apresenta a revisão sistemática da literatura das publicações que trataram do uso de SCA em empresas com foco na identificação de fatores motivadores e inibidores, formas de adoção e modelos de negócio praticados.

Os autores a seguir realizaram revisão de literatura em seus trabalhos, porém não da forma sistemática: [van Rooij 2009, Ven et al. 2008] buscaram fundamentação para elaborarem propostas de arcabouço para guiar o processo de seleção de ferramentas baseadas em SCA. [Bouras et al. 2012] estabeleceram comparações entre SCA e SP, destacando fatores técnicos, sociais, econômicos e organizacionais. Já o trabalho de [Payne and Singh 2010] pesquisou sobre uso de SCA em bibliotecas, destaque para funcionalidades, variedades de softwares, fatores motivadores e inibidores.

\section{Conclusão}

Este artigo apresentou uma revisão sistemática de literatura sobre a utilização de SCA pelas organizações, buscando identificar os fatores relatados como motivadores e inibidores, as formas de adoção e os modelos de negócio baseado em SCA.

O conhecimento dos fatores motivadores e inibidores, das diversas formas de adoção e dos modelos de negócios praticados podem auxiliar no desenvolvimento da estratégia de adoção de SCA pelas empresas, possibilitando mitigar os riscos da adoção. As estórias de sucesso são importantes para aumentar a confiança de empresa em softwares de código aberto [Glynn et al. 2005]. Mas não só o relato de sucesso é válido, o fracasso ou dificuldade também tem valor para quem tem interesse em adotar SCA.

Em concordância com os resultados de [Hauge et al. 2010], a presente revisão de literatura revelou que a pesquisa acadêmica sobre utilização de SCA em organizações é bastante fragmentada, carecendo de foco, rigor e estudos longitudinais. Principalmente na literatura nacional, as pesquisas se limitaram a apresentar estudos de casos, ora com foco em uma empresa, ora em um tipo específico de software.

\section{Referências}

Ayala, C., Cruzes, D. S., Hauge, O. y., and Conradi, R. (2011). Five Facts on the Adoption of Open Source Software. Software, IEEE, 28(2):95-99.

Bouras, C., Kokkinos, V., and Tseliou, G. (2012). SELECTING BETWEEN OPEN SOURCE AND PROPRIETARY SOFTWARE: THE PUBLIC ADMINISTRATORS'CASE. In IADIS International Conference e-Society, volume 2, pages 185-192.

da Silva, A. M. A. C., Mattos, C. V., Colugnati, F. A. B., and Veiga, R. (2005). Pesquisa Impacto do Software Livre e de Código Aberto (SL/CA) na Indústria de Software do Brasil : competências em SL / CA no Brasil. In XI Seminário Latino-Iberoamericano de Gestion Tecnologica, pages 1-14, Salvador/BA. 
Dipold, R. D. (2005). Potencialidade econômica do software livre. Monografia (bacharelado em ciências econômicas)-centro de ciências sociais, universidade estadual do oeste do paraná-campus de toledo, Universidade Estadual do Oeste do Paraná.

Fitzgerald, B. (2006). The Transformation of Open Source Software. MIS Quarterly, 30(3):587-598.

FLOSS (2002). Free/libre and open source software - survey and study. Technical report.

Glynn, E., Fitzgerald, B., and Exton, C. (2005). Commercial adoption of open source software: an empirical study. In Empirical Software Engineering, 2005. International Symposium on, pages 10-pp. IEEE, IEEE.

Goode, S. (2005). Something for nothing: management rejection of open source software in Australia?s top firms. Information \& Management, 42(5):669-681.

Hauge, O. y., Ayala, C., and Conradi, R. (2010). Adoption of open source software in software-intensive organizations ? A systematic literature review. Information and Software Technology, 52(11):1133-1154.

Hecker, F. (1999). Setting Up Shop : The Business of Open-Source Software. IEEE Software, 16(1):45-51.

Holck, J., Pedersen, M., and Larsens, M. (2005). Open source software acquisition: Beyond the business case. In European Conference on Information Systems. AIS.

Höst, M. and Oručević-Alagić, A. (2011). A systematic review of research on open source software in commercial software product development. Information and Software Technology, 53(6):616-624.

Kitchenham, B. (2004). Procedures for performing systematic reviews. Keele, UK, Keele University, 33.

Payne, A. and Singh, V. (2010). Open source software use in libraries. Library Review, 59(9):708-717.

Raymond, E. S. (1999). The Magic Cauldron.

Richter, D., Zo, H., and Maruschke, M. (2009). A comparative analysis of open source software usage in germany, brazil, and india. In International Conference on Computer Sciences and Convergence Information Technology, pages 1403-1410. IEEE.

Rusovan, S., Lawford, M., and Parnas, D. L. (2005). Open source software development: future or fad? Perspectives on Free and Open Source Software, pages 107-122.

Softex (2005). O impacto do software livre de código aberto na indústria de software do brasil. Technical report.

Stallman, R. (1999). The GNU Operating System and the Free Software Movement. In Open Sources: Voices from the Open Source Revolution, page 280. O'Reilly \& Associates Inc, 1 edition.

Stol, K.-J. and Ali Babar, M. (2010). Challenges in using open source software in product development: a review of the literature. In Proceedings of the 3rd International Workshop on Emerging Trends in Free/Libre/Open Source Software Research and Development, pages 17-22. ACM. 
Taurion, C. (2004). Software livre: potencialidades e modelos de negócio. Brasport, Rio de Janeiro.

Theunissen, W. H. M., Boake, A., and Kourie, D. G. (2004). A preliminary investigation of the impact of open source software on telecommunication software development. In Proceedings of the Southern African Telecommunication Networks and Applications Conference.

van Rooij, S. W. (2009). Adopting open-source software applications in us higher education: a cross-disciplinary review of the literature. Review of Educational Research, 79(2):682-701.

Ven, K., Van Grembergen, W., Haes, S. D., and Verelst, J. (2008). Using COBIT 4.1 to guide the adoption and implementation of open source software. Information Systems Control Journal, 3:31-35.

West, J. (2003). How open is open enough? Research Policy, 32(7):1259-1285.

West, J., Dedrick, J., and Place, B. (2005). The effect of computerization movements upon organizational adoption of open source. In Social Informatics Workshop: Extending the Contributions of Professor Rob Kling to the Analysis of Computerization Movements, pages 1-30, Irvine, California.

Wichmann, T. (2002). Use of open source software in firms and public institutions evidence from Germany, Sweden and UK. Technical Report July, Berlecon Research, Berlin, Germany.

Zhu, K. X. and Zhou, Z. Z. (2011). Lock-In Strategy in Software Competition: OpenSource Software vs. Proprietary Software. Information Systems Research, 23(2):536545.

Ziemer, S., Hauge, O. y., Ø sterlie, T., and Lindman, J. (2008). Understanding Open Source in an Industrial Context. In IEEE International Conference on Signal Image Technology and Internet Based Systems, pages 539-546. IEEE. 